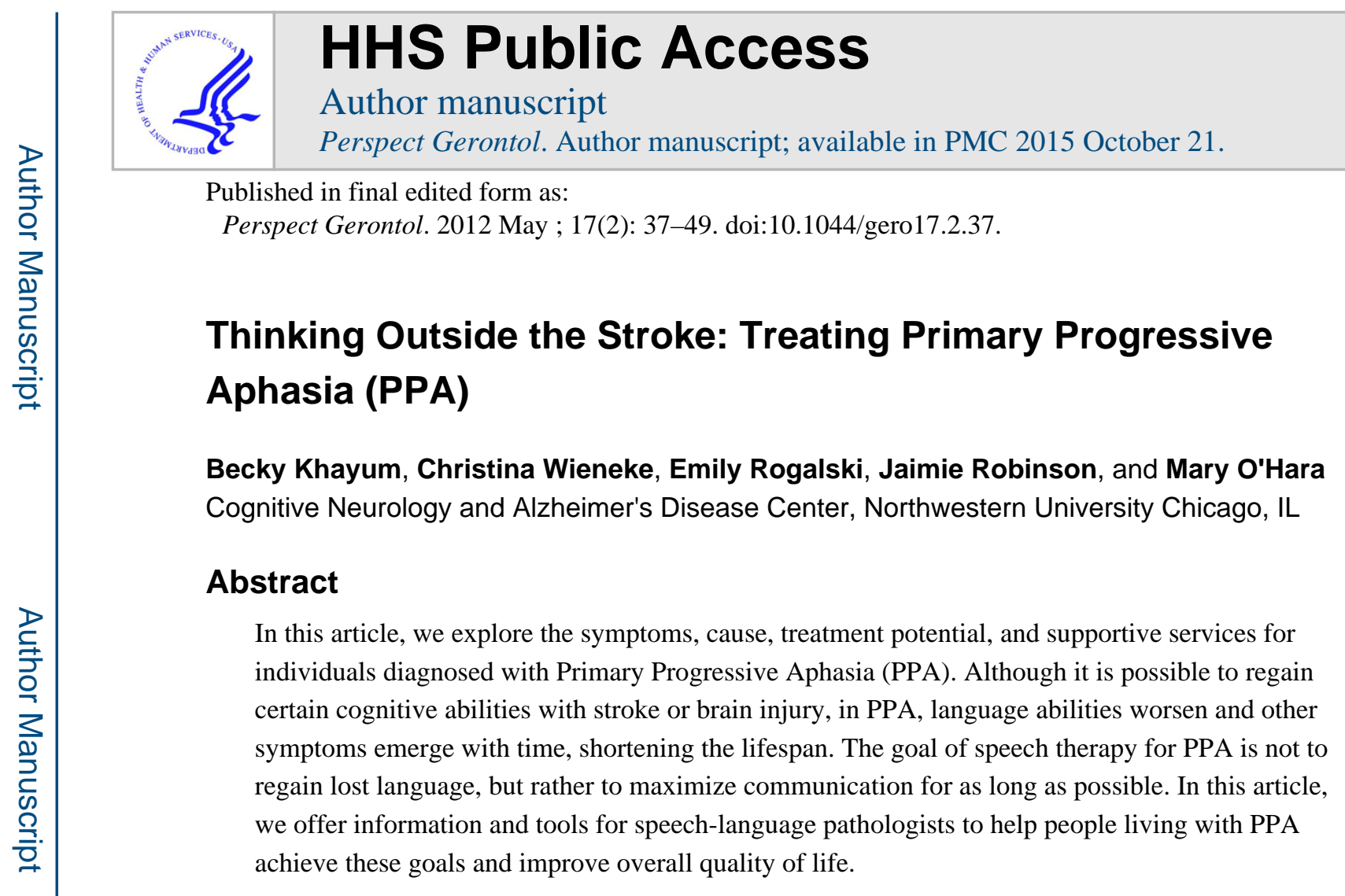

\title{
Understanding PPA
}

Primary Progressive Aphasia (PPA) is a relatively rare clinical dementia syndrome caused by neurodegenerative brain disease. Mesulam (1982) first described PPA, as "slowly progressive aphasia." Current diagnostic criteria (Gorno-Tempini et al., 2011) have defined PPA as a progressive dementia where language deficits are the primary presenting symptom. These deficits worsen over time and as the disease spreads to nonlanguage areas in the brain and affects other cognitive and behavioral functions. Although PPA can be diagnosed at any

RESOURCE LIST

Disease Information and Support

Northwestern University Cognitive Neurology and Alzheimer's Disease Center

www.brain.northwestern.edu

The International PPA Connection, IMPPACT

www.ppaconnection.org

Alzheimer's Disease Education and Referral Center (ADEAR) Publications

www.nia.nih.gov/alzheimers/publication

The Association for Frontotemporal Degeneration (AFTD)

www.theaftd.org

The National Aphasia Association (NAA)

www.aphasia.org

University of San Francisco Memory and Aging Center

www.memory.ucsf.edu

Future Care Planning

Social Security Disability

www.ssa.gov/disability/

National Association for Elder Law Attorneys

www.naela.org

Centers for Medicare and Medicaid Services

www.cms.gov/

Art, Music, and Dance Therapy

Each state has its own creative arts therapy associations. Search by state to find out about local art, music, and other creative therapies. 
age, the typical age of onset is between 40 and 60, classifying it as a "young-onset dementia."

It is important to differentiate PPA from aphasia associated with stroke or other brain injury.

The most salient difference lies in progression and the underlying disease. Although it is possible to regain abilities with stroke or brain injury, in PPA symptoms worsen and other symptoms emerge with time, foreshortening the lifespan.

The cause of PPA remains elusive and it is rarely hereditary $(<10 \%)$ although some familial cases have been reported (Mesulam et al., 2007). The clinical syndrome of PPA is associated with two main classes of neuropathology discovered only after the patient dies, upon postmortem brain autopsy: Alzheimer's disease (AD) and frontotemporal lobar degeneration (FTLD; Mesulam et al., 2008).

Currently, there are no effective or FDA approved medications to treat PPA. Anecdotal reports indicate that speech therapy may be useful, particularly if the therapy is provided throughout the course of the disease. The goal of speech therapy for PPA is not to regain lost language ability, but rather to maximize communication ability for as long as possible.

In this article, we provide an overview of the current understanding of the syndrome and the importance of nonpharmacologic treatment options for improving quality of life. In particular, we highlight the potential role that the speech-language pathologist (SLP) may play and provide practical recommendations for how one might integrate speech therapy throughout the disease course.

\section{Variants and Progression of PPA}

Recent research has led to progress in understanding the clinical characteristics, genetics, and neuropathology of PPA. Experts generally recognize three clinical variants of the syndrome: agrammatic (PPA-G), semantic (PPA-S), and logopenic (PPA-L). Recently published consensus criteria outline the diagnostic guidelines for these variants (GornoTempini et al., 2011). PPA-G is most often associated with agrammatism in language production and/or labored, effortful speech. Additionally, individuals with this type of PPA often have impaired comprehension of syntactically complex sentences but spared singleword comprehension. For example, they may have difficulty understanding who pushed whom in the sentence "It was the girl who the boy was pushing." Individuals with PPA-S have severe object anomia and impaired single-word comprehension, particularly for lowfamiliarity nouns (e.g., accordion). They may display surface alexia or agraphia and impaired object knowledge. Individuals with PPA-S typically have spared speech production (grammar and motor speech), but sentences are often tangential or vague and lacking nouns. The hallmark of PPA-L is impaired retrieval of single words during naming tasks and in conversation. Individuals with this variant also may demonstrate impaired repetition of sentences and phrases. They usually have spared single-word comprehension and speech production (motor speech and grammar) but may make phonological errors when naming. People with PPA-L may seem fluent in casual conversation through use of circumlocution and automatic phrases, but become dysfluent when forced to be specific. Because

Perspect Gerontol. Author manuscript; available in PMC 2015 October 21. 
dysfunction in PPA can begin in one of several components within the language network, individuals can show features of multiple subtypes (i.e., both grammar and single-word comprehension impairment) and are diagnosed with "mixed" PPA. (Mesulam et al., 2009)

Subtyping is easiest at the mild to moderate stages, but is challenging for the nonexpert. Caused by neurodegenerative disease, deficits in PPA worsen over time and the boundaries between subtypes become more difficult to differentiate. As an SLP, however, identifying the primary deficit is more important than the nomenclature. For example, it is important to distinguish between someone who has trouble thinking of words in conversation and one who no longer remembers the meaning of words. The former may benefit from self-cueing strategies, while the latter may not.

Because the disease is progressive, functional decline inevitably occurs, however, the rate of decline is variable from person to person. Estimates of life expectancy average 7-10 years after diagnosis, but researchers have observed rare cases of 23-year duration (Mesulam et al., 2008). It is unclear why some progress more rapidly than others. Although language always worsens as the disease advances, deficits in other cognitive abilities eventually emerge. When language is no longer the most salient aspect of PPA, researchers use the term "PPA+" to indicate that symptoms include deficits in other cognitive domains (e.g., memory, attention, judgment, changes in behavior and personality; Rogalski \& Mesulam, 2009). This presents a unique challenge to SLPs because these additional changes must be factored into compensatory strategies for communication. Figure 1 describes possible symptom progression in variants of PPA. PPA-G and PPA-S are associated with problems with executive function and changes in personality, respectively. However, becarse PPA-L was defined recently (Gorno-Tempini et al., 2004), much less is known about symptom progression of this variant. It is important to note that not every person with PPA experiences this sequence of decline. The temporal order of language decline and emergence of deficits in cognitive domains other than language may differ from person to person and among subtypes. For example, whereas behavior and personality changes are more common in PPA-S, other variants may experience these symptoms as well. In addition, swallowing problems may emerge early or late in the course of disease. Therefore, treatment plans need to adapt to each person's needs over time.

Although a neurologist typically makes the diagnosis of PPA with supportive documentation from brain imaging and other evaluations, an SLP is an integral part of the care team that also includes neuropsychologists, social workers and psychiatrists. In the absence of medical treatments for PPA, speech and language therapy is essential to maintain and maximize the patient's communication ability.

\section{PPA and the Role of the SLP}

Treatment of PPA must be carefully differentiated from treatment of aphasia after stroke. Many SLPs may be tempted to quickly discharge an individual with PPA because the condition continuously worsens and it is difficult to document progress towards traditional comprehension and production language goals. However, SLPs are uniquely trained not only to provide treatment-focused compensation strategies, but also to connect the individual 
with the necessary resources and provide much needed counseling. Caregiver/family education and training, important for any diagnosis, is critical in treating those with PPA. An SLP always should review strategies for effective communication with the family as well as the diagnosed individual. Also, the plan of treatment will change over the course of the disease and knowing how to develop functional, patient-centered goals is key to helping individuals with PPA communicate as independently as possible. An SLP should also clearly define appropriate therapy goals to ensure reimbursement for services.

\section{Evaluation of PPA}

Outside of a research setting, standardized tests likely are not the best method for determining appropriate treatment strategies for an individual with PPA. They certainly are not helpful in documenting progress for reimbursement purposes because most individuals do not demonstrate improved scores on tests such as the Western Aphasia Battery (WAB) after treatment. Although test scores may actually decrease, it does not mean the individual has not benefited. Instead, progress should be measured toward individualized, functional communication goals. The SLP should write goals that the individual will be able to achieve, even if moderate or maximal cueing is needed. He or she should include the specific task that the goal is targeting (e.g., giving a class lecture or ordering at a restaurant). If an individual is not progressing towards their goals in the plan of care, then it is the SLP's role to re-examine the goals to determine if they are appropriate.

One tool to help set appropriate goals for persons with PPA is the Progressive Aphasia Severity Scale (Sapolsky et al., 2010). SLPs can use the scale for a systematic approach without using a standardized test. The scale includes severity ratings for articulation, fluency, syntax and grammar, word retrieval and expression, repetition, auditory comprehension, single word comprehension, reading, writing, and overall functional communication.

Throughout the evaluation, the SLP should complete informal assessment of expressive and receptive language, speech production, writing, reading, and cognition, with a focus on trialing treatment strategies. During the evaluation, the SLP should consider the following questions.

\section{All Stages}

- What kind of caregiver support is available? What adaptations has the family already made to accommodate the person? How will I incorporate the family into speech therapy?

- Is the individual aware of their impairment and motivated to work on communication strategies?

- What is the family's understanding of the diagnosis? Does the family need counseling to cope with the diagnosis? Is there a need for psychiatry or social work?

- Does the person have memory loss? Will this influence the generalization of treatment strategies?

Perspect Gerontol. Author manuscript; available in PMC 2015 October 21. 
- How is the condition affecting the ability to complete activities of daily living (ADLs)? What strategies can enable the individual to complete these independently?

- What strategies can I use to help the individual maintain his/her identity?

Mild Stage

- What self-cueing strategies is the person already using (e.g., semantic elaboration, writing)?

- What self-cueing strategies can the individual use with minimal prompting for initiation?

- Is the individual a good candidate for script training?

- Is the individual still working? Are there any compensatory strategies that will allow the individual to work longer?

\section{Moderate and Severe Stages}

- Are the person and family open to a Communication Book or AAC device options? Are these realistic in terms of motivation and capacity to use these? Often, it is useful to introduce these strategies earlier in the course of illness when the individual may not need them but can learn to depend of them for later in the course of illness.

- What strategies is the individual currently using to communicate? Have alternate modes of communication already been established?

- How much training has the family had in promoting the use of alternate modes of communication?

\section{Treatment Strategies for PPA}

Although there has been little research to date specifically focusing on language treatment for PPA, there is a small but growing body of evidence indicating that individuals with PPA may demonstrate acute improvement in language in response to speech and language treatment, particularly in the early stages. More research is needed to assess generalization and durability of improvements over time.

Often, the traditional strategies SLPs use to facilitate language in stroke aphasia are not effective in treating PPA. Instead, when working with this population, SLPs should focus on specific compensatory strategies that will lead to an improvement in functional communication in the home and possibly work environments.

It is not appropriate to "graduate" an individual with PPA. Rather, these individuals will need intervention for short periods of time throughout the disease course so that SLPs can make new recommendations, train the family on new strategies, and introduce alternate modes of communication when necessary. SLPs should avoid homework or drills that frustrate the individual with PPA. If the person has no desire to make telephone calls to 
make appointments and has a family member that is able to do this task, this is not an appropriate treatment goal.

\section{Promoting Self-Cueing Strategies}

In the mild stages of PPA, SLPs should focus on self-cueing strategies for lexical retrieval, which capitalize on the individual's residual skills. Semantic, orthographic, and phonological self-cueing all should be assessed during the evaluation in order to determine which strategy is most successful in prompting retrieval. Family members can be trained to provide the appropriate cueing strategies for initiation of self-cueing such as "Tell me about it" or "Can you think of what letter it starts with?" The eventual goal is to have the individual begin to spontaneously self-cue at the conversation level (Beeson, 2011). However, the SLP also needs to be mindful of when the family member is working too hard or using all interactions as therapy sessions.

\section{Script Training}

Researchers have documented the benefits of conversational scripts to increase functional communication in stroke-induced aphasia (Youmans, Holland, Muñoz, \& Bourgeois, 2005; Youmans, Youmans, \& Hancock, 2011). The benefit of script training lies in the automaticity of skills patients gain by retrieving memories of context-bound, skilled performance (Logan, 1988). Repeated practice of a specific task can enhance automatic retrieval during a functional situation when the same stimuli from the practice environment are present. The key to script training is ensuring that the individual practices consistently and intensively (Cherney, 2011).

When creating a script, the SLP should consider the individual's needs and interests, the type of script (dialogue or monologue), the number and length of conversational turns, and its grammatical complexity and vocabulary selection (Cherney, 2011). Families may purchase a script computer program such as AphasiaScripts.

Examples of possible script topics include:

- Talking to a family member or friend (e.g. by phone)

- Scheduling an appointment; asking for directions

- Stating personal information or explaining PPA

- Giving a lecture or speech

- Saying prayers

SLPs can be write goals based on the accuracy and rate of production (e.g., timing how long it takes to read the script while counting the number of errors). If the person is not motivated to practice the script outside of therapy sessions, he or she may not be a good candidate for script training. 


\section{Facilitating Receptive Language}

For individuals exhibiting deficits in receptive language, family training on strategies to facilitate comprehension is essential. Communication partners can make a difference in comprehension by automatically

- speaking slowly, facing the person and ensuring you have full attention,

- using gestures to supplement speech,

- giving one direction at a time,

- using simple grammatical structure and vocabulary, or

- eliminating distractions (e.g., turning off the TV/radio) during important conversations.

\section{Low-Tech AAC}

It is usually appropriate to begin discussing alternate modes of communication in the moderate stages of the disease (or possibly earlier if the person has motor-speech problems). These alternate modes of communication are not meant to discourage the individual from trying to speak, but rather as a way to supplement communication as well as a "back up" option during a communication breakdown. SLPs should counsel families about their need for an alternate form of communication as the disease progresses and verbal output becomes limited.

A communication book is an excellent low-tech AAC option. The best communication books are those that are completely catered to the individual's personal needs and interests. The SLP's role is to assess the appropriate format, font size, and word/phrase length for the book and then to train the individual and family members to continue adding pages.

SLPs should consider a number of factors when forming a communication book.

- SLPs can laminate small pictures, so the patient can place them on a key ring and easily carry them in a pocket.

- For a more comprehensive book, SLPs should use plastic page protectors in a binder.

- SLPs can individualize pages by using images from the Internet or the person's own photographs.

- SLPs can use tabs to divide the book into sections (see Figure 2 for suggested sections; see www.brain.northwestern.edu for an example book).

- SLPs should ask family members to record words and ideas that have led to communication breakdowns. They should add these to the book.

Because some individuals with PPA may not independently use the book, training the family members on appropriate cueing strategies to initiate book use and facilitate navigation also should be targeted during treatment sessions.

Perspect Gerontol. Author manuscript; available in PMC 2015 October 21. 


\section{High-Tech AAC Devices}

Although there is no research documenting AAC intervention strategies for persons with PPA, SLPs have successfully used high-tech AAC devices to facilitate communication in those with severe aphasia caused by stroke (Beukelman, Fager, Ball, \& Dietz, 2007). Because other cognitive abilities will worsen over time in people with PPA, it is important to consider that a person's ability to learn to use a high-tech device may be limited and eventually will be impaired.

It is the SLP's role to determine which, if any, of the devices will help the individual best communicate his or her needs and allow for communication in social environments. When evaluating if a high-tech AAC is a good fit for someone with PPA, the SLP should ask a number of questions.

- Is the person motivated and interested to use a high-tech ACC to communicate? If not, it is not likely to be successful.

- Can the person operate other types of technology like a computer, microwave, TV remote, or telephone independently? If not, this could indicate a loss of fine motor skills or inability to sequence and demonstrates a poor fit for an ACC.

- Has the family member(s) been involved in the set-up, training, and goals? If not, invite family members to multiple sessions to insure success once implemented.

- Have the person and their family been trained to use the device in normal environments? Have the trials met set goals? If not, consider more training and an extensive trial.

Note that Medicare will only pay for some high-tech devices if the beneficiary meets certain requirements. Private insurance may or may not cover part of the cost of certain devices. Due to the high cost of these devices, it is important to explore out-of-pocket cost and insurance coverage before considering such a device.

\section{Case Study: TM}

\section{Setting: Outpatient Clinic}

TM was a 65-year-old male. His spouse began noticing changes in his speech 3 and a half years ago, he often used the wrong words and had slow responses during conversation. After neuropsychological testing and examination by a neurologist, he was diagnosed with PPA. His aphasia quotient from the WAB was 86 . When he began having difficulty in his position as a college professor, he was forced to retire. His symptoms progressively worsened, it became more difficult for him to complete multistep tasks and stay organized. His spouse also reported that he had difficulty concentrating, experienced emotional outbursts, and perseverated on topics, such as eating.

TM reported that he was frustrated by these changes in cognition and was worried about losing his independence. His spouse worked full-time, so he was home alone during the day and became easily bored, spending most of his time watching TV. Most importantly, he felt that he was losing his identity, as his passion had been to teach others. In addition to

Perspect Gerontol. Author manuscript; available in PMC 2015 October 21. 
teaching, he used to mentor students from his fraternity. He considered volunteering, but worried the activity would rely too heavily on language.

We completed an informal assessment of language production and found moderate communication deficits. His speech was nonfluent and he exhibited frequent hesitations for word retrieval and semantic paraphasias. Remarkably, he had preserved repetition at the word, phrase, and sentence levels, making him an excellent candidate for a speechgenerating device that would allow him to program his own speech. He experienced only mildly impaired confrontation-naming for common objects. TM demonstrated the ability to use semantic circumlocution at the conversation level with minimal verbal cues, although he reported that this strategy became increasingly difficult as his speech worsened over the prior few months. He also exhibited moderate alexia at the paragraph level and severe agraphia at the word level. Researchers noted that oral reading at the paragraph level improved significantly after several trials in terms of fluency and word retrieval, making him an excellent candidate for script training. His receptive language was mildly-moderately impaired, with significant difficulty following multistep commands (0/3, apraxia noted), answering complex yes/no questions (0/3), and comprehending conversation, especially if sentence complexity or speaking rate was increased.

\section{Recommendations}

Staff recommended skilled speech therapy once a week for 6 weeks along with expressive language treatment, focusing on compensatory strategies to aide functional communication.

Staff provided a number of treatment recommendations for the patient (TM) and his spouse (S).

- Train the increased use of semantic circumlocution for self-cueing at the conversation level to decrease communication breakdowns with family and friends. (TM)

- Create functional scripts for the telephone, social settings, and mentoring fraternity students. TM will rehearse the scripts for 30 minutes/day. (TM and S)

- Create a communication book to be used to supplement speech when needed. (TM and $S$ )

- Try the GoTalk20 AAC device, which will allow TM to program his own speech. The trial includes practicing with this device and using it to speak over the telephone. (TM)

- Try some volunteer activities that don't require a great deal of talking, such as a food pantry. TM was connected with a professor in the Speech-Language Pathology department at a nearby university who arranged for him to speak to her Neurogenic Speech Disorders class about his condition using a script. (TM)

- Get involved in an aphasia support group at the university (TM and S) 


\section{Short-Term Goals}

1. TM will demonstrate the use of semantic circumlocution during structured conversation to retrieve words or communicate his message with $80 \%$ accuracy and minimal verbal cues to increase functional communication with spouse, family members, and friends.

2. TM will read a telephone script during structured tasks, with $<3$ errors and in $<2$ minutes, 30 seconds with minimal gestural cues to increase ability to communicate with his mother over the telephone. (Baseline: 8 errors, 3 minutes)

3. TM will correctly navigate to $9 / 10$ functional icons in the communication book, given minimal verbal and gestural cues, to increase ability to communicate with spouse in the home environment.

4. TM will independently initiate use of the communication book 3 times during the session to increase his ability to communicate needs with spouse and medical staff.

5. TM will repeat 3-5 word sentences with $90 \%$ accuracy in order to enter speech into an AAC device to increase ability to communicate needs at home and in the community.

\section{Long-Term Goal}

TM will communicate his needs and communicate in social environments using word retrieval strategies, conversational scripts, a Communication Book, and an AAC device with $>90 \%$ accuracy and minimal cues from his spouse to increase safety and independence at home and in the community.

\section{Every PPA Patient is Different}

SLPs must remember that every person with PPA is different. A strategy that works for one person may not work for another. An SLP's goal should not be to regain lost language function, but rather to put strategies in place so that the person is able to communicate their most basic needs and thoughts in the most effective way possible, for as long as possible. It is necessary for SLPs to think outside of the box and continuously adapt treatment plans. Although a person with PPA may not "improve," the communication skills taught by an SLP improve the quality of life for both the diagnosed person and their family.

\section{Quality of Life Interventions}

Until there is a cure for PPA, SLPs and other professionals must help individuals and families living with a diagnosis cope with changes and find interventions to enhance a patient's quality of life.

Quality of life interventions are nonpharmacological (nonmedical) approaches to care that focus on providing meaningful activity, enhancing coping skills, and addressing changes in mood that often accompany a dementia diagnosis. 
A diagnosis of PPA means not only a progressive loss of language, but also a progressive loss of a patient's abilities in many areas that affect his or her life. Many individuals are working at the time of their diagnosis and are at a prime-earning point in their careers. However, as the disease progresses, the symptoms become too severe and parents can no longer work, affecting the financial stability of the family. Other significant life changes include changes in relationships and roles within the family and, when the disease progresses, the loss of markers of autonomy such as driving.

Although a small proportion of people with PPA do not have insight into their condition, most diagnosed persons are very aware of their loss of language ability. Combined with social withdrawal and loss of independence, about one-third of people with PPA experience clinically significant depression and many more experience depressive symptoms in the subclinical range (Medina \& Weintraub, 2007). As part of caring for individuals with PPA, SLPs should arrange for ongoing clinical assessments for depression and ensure that treatment such as counseling, medications, and involvement in meaningful activity are offered.

\section{Disease Education}

Because PPA can be difficult to understand, knowledge about the symptoms, progression, and where to turn to for help is essential for both the diagnosed individual and his or her family. Although it is important that family members understand the progressive nature of PPA, it is equally important they are aware of the options for care that focus on promoting independence and quality of life. For example, supportive services like occupational therapy and physical therapy can promote independence and safety in the early stages and as the disease progresses. The NIH has published a booklet for individuals with PPA and other clinical disorders that fall under the rubric of frontotemporal lobar degeneration (see Resource List).

\section{Future Planning}

Families should discuss long-term care planning soon after the diagnosis. This allows the diagnosed individual to make his or her wishes known and to participate in the plan for his or her future care, as the patient will be unable to participate in these kinds of decisions as communication and cognition become more impaired. Although these conversations can be difficult, having them early provides family members and health care providers with invaluable guidance about a person's wishes for care and other decision-making.

As part of future planning, diagnosed individuals should establish a Power of Attorney for health care and finances (Robinson et al., 2011). Individuals under the age of 65 should apply for Social Security Disability Insurance (SSDI). PPA is on the Compassionate Allowance list for SSDI, ensuring those who apply are automatically approved. After 2 years, people with SSDI are eligible for Medicare.

\section{Emotional Support}

Family members and individuals living with PPA may benefit from additional emotional support such as individual therapy or support groups (Kaiser \& Panegyres, 2007). Although

Perspect Gerontol. Author manuscript; available in PMC 2015 October 21. 
involvement in a PPA specific support group is ideal, these programs are limited in their availability because it is a less common form of dementia.

\section{Finding Meaning}

Because of the numerous losses that individuals with PPA endure, it is critical that these patients find ways of staying connected and engaged in meaningful activity. SLPs should work with the patient and family to modify previous hobbies to fit current functional and cognitive abilities in order to minimize isolation and to ensure that the individual continues to stay mentally engaged. For example, a person who enjoyed volunteering at a community center could continue to do this with the understanding that tasks must be continually modified to meet the person's abilities (Mahendra \& Arkin, 2004).

Creative arts also offer enriching experiences for people with dementia (Brotons \& Koger, 2000). Creative art therapies allow people who are losing their ability to communicate verbally to express themselves emotionally through art, music, or movement (Phillips, ReidArndt, \& Pak, 2011).

\section{Conclusion}

Although people with PPA have a progressive and neurodegenerative illness, SLPs should not shy away from treating patients diagnosed with this rare condition. The education and strategies outlined in this article provide good starting points for assessment and treatment. The SLP is an integral part of the care team and can offer diagnosed persons and their family a positive outlook on an otherwise devastating condition.

\section{Acknowledgments}

Thank you to Darby Morhardt, LCSW, and Sandy Weintraub, PhD for their contributions to this article.

\section{Biography}

Becky Khayum is a speech-language pathologist who specializes in the treatment of cognitive-communication disorders. She is the co-founder and president of MemoryCare Corporation, a company dedicated to providing individualized therapy services to those suffering from Alzheimer's disease and other memory disorders. She also specializes in the evaluation and treatment of Primary Progressive Aphasia. Prior to her work with MemoryCare, Becky provided services in assisted living communities and skilled nursing facilities around the Chicago region. Becky has developed a passion for educating professionals and caregivers on strategies that facilitate communication and memory for individuals with various forms of dementia. Becky completed her graduate studies at the University of Arizona and undergrad at Purdue University.

Christina Wieneke, BA, is a research project manager at the Northwestern University Cognitive Neurology and Alzheimer's Disease Center (CNADC). At the CNADC, she manages all components of the longitudinal, NIH-funded Language in Primary Progressive Aphasia research program, including subject recruitment, neuropsychological evaluation,

Perspect Gerontol. Author manuscript; available in PMC 2015 October 21. 
and disease education. She received a BA from Purdue University in behavioral neuroscience.

Emily J. Rogalski, PhD, is currently the Assistant Research Professor at Northwestern University's Cognitive Neurology and Alzheimer's Disease Center. She has more than 10 years of experience in the field of aging and dementia, specializing in primary progressive aphasia (PPA) research. Dr. Rogalski has assisted in developing educational programs and support groups for caregivers, family members, and individuals with PPA. She is an author of several articles that provide insight into brain-behavior relationships, risk factors, and perspective about the clinico-anatomical progression over time in PPA. The outcomes of her work have translational value in providing recommendations for patient care.

Jaimie Robinson, MSW, LCSW, is currently the Resource Navigator at the Neurobehavior and Memory Clinic at Northwestern University's Cognitive Neurology and Alzheimer's Disease Center, where she guides the person diagnosed with a form of dementia and their family to cope with the cognitive symptoms. Jaimie received her Master's in Social Work from Loyola University Chicago, after which she worked at the Alzheimer's Association National Office in Family Programs. Jaimie has a special interest in the needs of persons who are aging, particularly those who are living with cognitive changes. Jaimie is a featured writer and manager of a professional blog called the Chicago Bridge and is a member of the board of directors for Chicago's Society of Social Worker Leadership in Health Care.

Mary O'Hara, AM, LCSW, is a social worker and the Assistant Director of Education at Northwestern's Cognitive Neurology and Alzheimer's Disease Center (CNADC). In addition to providing education and outreach to the community and facilitating the CNADC's various Quality of Life Enrichment Programs, Mary also facilitates family caregiver and diagnosed individual support groups. In the Neurobehavior and Memory Clinic, Mary provides education and support to diagnosed persons and their families. Mary received her master's degree from The University of Chicago's School of Social Service Administration, where she focused on working with older adults.

\section{References}

AphasiaScripts [Computer software]. Rehabilitation Institute of Chicago; Chicago, IL:

Beeson, P. Updates in aphasia rehabilitation for the practicing speech-language pathologist.; Presented at the Rehabilitation Institute of Chicago, Chicago, IL. 2011, June;

Beukelman DR, Fager F, Ball L, Dietz A. AAC for adults with acquired neurological conditions: A review. Augmentative and Alternative Communication. 2007; 23:230-242. [PubMed: 17701742]

Brotons M, Koger S. The impact of music therapy on language functioning in dementia. Journal of Music Therapy. 2000; 37:183-195. [PubMed: 10990596]

Cherney, L. Updates in aphasia rehabilitation for the practicing speech-language pathologist.; Presented at the Rehabilitation Institute of Chicago, Chicago, IL. 2011, June;

Gorno-Tempini ML, Dronkers NF, Rankin KP, Ogar JM, Phengrasamy L, Rosen HJ, Miller BL. Cognition and anatomy in three variants of primary progressive aphasia. Annals of Neurology. 2004; 55:335-346. [PubMed: 14991811]

Gorno-Tempini ML, Hillis AE, Weintraub S, Kertez A, Mendez M, Cappa S, Grossman M. Classification of primary progressive aphasia and its variants. Neurology. 2011; 76:1006-1014. [PubMed: 21325651] 
Kaiser S, Panegyres P. The psychosocial impact of young onset dementia on spouses. American Journal of Alzheimer's Disease and Other Dementias. 2007; 21:398-402.

Logan GD. Toward an instance theory of automatization. Psychological Review. 1988; 95:492-527.

Mahendra N, Arkin S. Exercise and volunteer work: Contexts for AD language and memory interventions. Seminars in Speech and Language. 2004; 25:151-167. [PubMed: 15118942]

Medina J, Weintraub S. Depression in primary progressive aphasia. Journal of Geriatric Psychiatry and Neurology. 2007; 20:153-160. [PubMed: 17712098]

Mesulam MM. Slowly progressive aphasia without generalized dementia. Annals of Neurology. 1982; 11:592-598. [PubMed: 7114808]

Mesulam M, Johnson N, Krefft TA, Gass JM, Cannon AD, Adamson JL, Graff-Radford NR. Progranulin mutations in primary progressive aphasia: The PPA1 and PPA3 families. Archives of Neurology. 2007; 64:43-47. [PubMed: 17210807]

Mesulam M, Wicklund A, Johnson N, Rogalski E, Léger GC, Rademaker A, Bigio EH. Alzheimer and frontotemporal pathology in subsets of primary progressive aphasia. Annals of Neurology. 2008; 63:709-719. [PubMed: 18412267]

Mesulam M, Wieneke C, Rogalski E, Cobia D, Thompson C, Weintraub S. Quantitative template for subtyping primary progressive aphasia. Archives of Neurology. 2009; 66:1545-1551. [PubMed: 20008661]

Phillips L, Reid-Arndt S, Pak. Y. Effects of creative expression intervention on emotions, communication and quality of life in persons with dementia. Nursing Research. 2010; 59:417-425. [PubMed: 21048483]

Robinson L, Dickinson C, Rousseau N, Beyer F, Clark A, Hughes J, Exley C. A systematic review of effectiveness of advance planning interventions for people with cognitive impairment and dementia. Age and Ageing. 2011; 41:263-269. [PubMed: 22156555]

Rogalski EJ, Mesulam MM. Clinical trajectories and biological features of primary progressive aphasia (PPA). Current Alzheimer's Research. 2009; 6:331-336.

Sapolsky D, Bakkour A, Negreira A, Nalipinski P, Weintraub S, Mesulam MM, Dickerson BC. Cortical neuroanatomic correlates of symptom severity in primary progressive aphasia. Neurology. 2010; 75:358-366. [PubMed: 20660866]

Youmans GL, Holland AL, Muñoz M, Bourgeois M. Script training and automaticity in two individuals with aphasia. Aphasiology. 2005; 19:435-450.

Youmans G, Youmans SR, Hancock AB. Script training treatment for adults with apraxia of speech. American Journal of Speech-Language Pathology. 2011; 20:23-27. [PubMed: 20739633]

Perspect Gerontol. Author manuscript; available in PMC 2015 October 21. 
PPA

Language continues to get worse PPA +
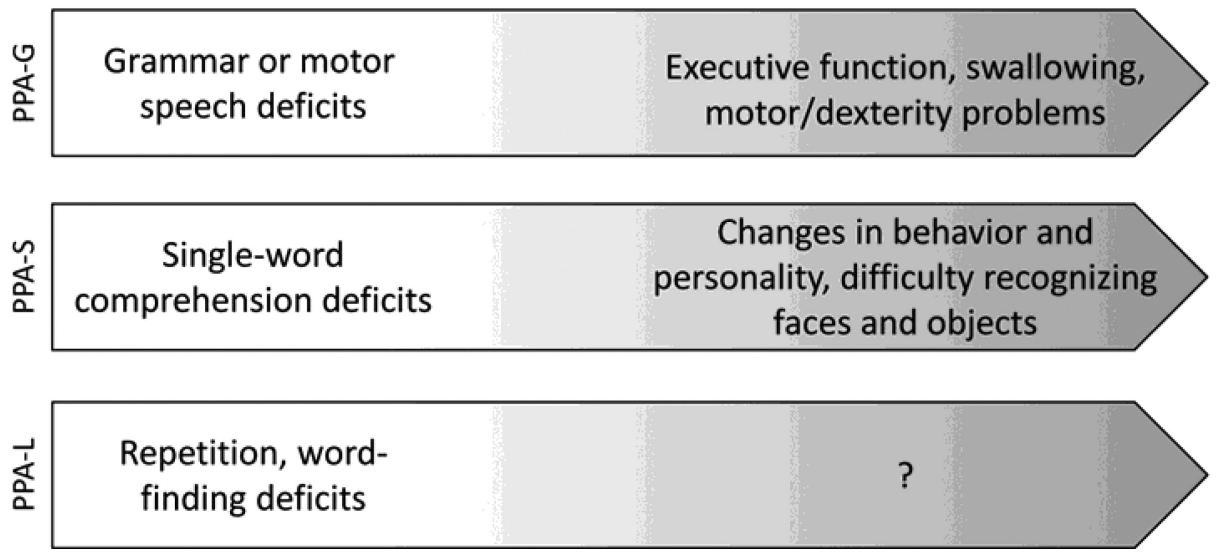

Figure 1.

The Progression of Aphasia 


\section{Possible Sections in Communication Books}

Personal/biographical facts (include old photographs)

Personal history: occupation, places lived, etc.

Family and friends (use pictures and list contact information)

Hobbies

Everyday objects around the home

Clothing

Food and beverages (divide by meal)

Health issues (for communicating medical needs; include an explanation of PPA)

Pages specifically for communicating with specific family members or friends (e.g. grandchildren)

Favorite sports, restaurants, recipes, etc.

Places traveled (with photos)

Frequently visited stores and typical items on a grocery list

Figure 2.

Possible Sections in Communications Books 